\title{
Transporter engineering for improved tolerance against alkane biofuels in Saccharomyces cerevisiae
}

\author{
Binbin Chen, Hua Ling and Matthew Wook Chang ${ }^{*}$
}

\begin{abstract}
Background: Hydrocarbon alkanes, components of major fossil fuels, are considered as next-generation biofuels because their biological production has recently been shown to be possible. However, high-yield alkane production requires robust host cells that are tolerant against alkanes, which exhibit cytotoxicity. In this study, we aimed to improve alkane tolerance in Saccharomyces cerevisiae, a key industrial microbial host, by harnessing heterologous transporters that potentially pump out alkanes.
\end{abstract}

Results: To this end, we attempted to exploit ABC transporters in Yarrowia lipolytica based on the observation that it utilizes alkanes as a carbon source. We confirmed the increased transcription of $A B C 2$ and $A B C 3$ transporters upon exposure to a range of alkanes in Y. lipolytica. We then showed that the heterologous expression of $A B C 2$ and $A B C 3$ transporters significantly increased tolerance against decane and undecane in S. cerevisiae through maintaining lower intracellular alkane level. In particular, $A B C 2$ transporter increased the tolerance limit of $S$. cerevisiae about 80 -fold against decane. Furthermore, through site-directed mutagenesis for glutamate (E988 for $A B C 2$, and $E 989$ for $A B C 3)$ and histidine ( $\mathrm{H} 1020$ for $A B C 2$, and $H 1021$ for $A B C 3)$, we provided the evidence that glutamate was essential for the activity of $A B C 2$ and $A B C 3$ transporters, with ATP most likely to be hydrolyzed by a catalytic carboxylate mechanism.

Conclusions: Here, we demonstrated that transporter engineering through expression of heterologous efflux pumps led to significantly improved tolerance against alkane biofuels in S. cerevisiae. We believe that our results laid the groundwork for developing robust alkane-producing yeast cells through transporter engineering, which will greatly aid in next-generation alkane biofuel production and recovery.

Keywords: Biofuels, ABC transporters, S. cerevisiae, Alkanes, Tolerance

\section{Background}

The development of renewable biofuels such as bioethanol [1], butanol [2], bio-diesel [3-5] and jetfuels [6] helps to address energy security and climate change concerns. Recently, the biological production of hydrocarbon alkanes, components of major fuels, has drawn much attention because alkanes have high energy content and are compatible with existing transportation infrastructure. In addition, alkanes have been widely used as an organic solvent in biochemical processes in an effort to improve substrate solubility, enzyme stability and

\footnotetext{
* Correspondence: Matthewchang@ntu.edu.sg

Division of Chemical and Biomolecular Engineering, School of Chemical and Biomedical Engineering, Nanyang Technological University, 62 Nanyang Drive, Nanyang 637459, Singapore
}

specificity $[7,8]$. In nature, alkanes are found to be produced from fatty acid metabolites in microorganisms [9,10], insects [11] and plants [12]. Recently, an alkane biosynthetic pathway was identified and characterized in cyanobacteria and re-constructed in Escherichia coli $[10,13]$. In addition, plant alkane biosynthesis pathway was reconstituted in yeast [14].

Despite this promising potential of microbial alkane production, yields and titers are key consideration for industrial-scale production. Further, alkane biofuel production can potentially be affected by the product toxicity as alkanes are proven to be toxic to microorganisms $[15,16]$. The toxicity of products can be evaluated based on $\log P_{o w}$ which represents the logarithm of partition coefficients in n-octanol and water [17]. Organic products 
with a $\log P_{o w}$ between 1.5 and 6.0 are extremely toxic for microorganisms and other living cells, such as nonane $\left(\log P_{\text {ow }}=5.5\right)$ and decane $(\log P$ ow $=6.0)$ [18]. These alkane products interact preferentially with cytoplasmic membrane, therefore disorganizing its structural integrity. Disruption of membrane structure impairs vital functions, such as the loss of ions, metabolites, lipids, and proteins, and the dissipation of the $\mathrm{pH}$ gradient and electrical potential. In addition, several studies have shown that tolerance improvement can lead to clear increases in biofuel production [19-21]. Consequently, there is an urgent need to develop robust microbial cell factories that are tolerant to alkane biofuels.

To overcome biofuel toxicity, several engineering strategies have been designed. Alper et al. [19] employed a global transcription machinery engineering (gTME) approach to improve ethanol tolerance. Stanley et al. [22] used an adaptive evolution engineering method to select stable ethanol-tolerant mutants of $S$. cerevisiae. Hou et al. [23] developed novel genome shuffling method to improve biofuel tolerance, whereas Kang et al. [24] improved microbial tolerance to isooctane through identification and reconstitution of genetic regulatory networks. However, these classical tolerance-improving strategies can be time consuming and laborious. Recently, efflux pumps were utilized to alleviate product toxicity and increase final productivity because of their capability to pump out target products from cells $[21,25]$. However, no attempts have been made to exploit efflux pumps for alkane biofuel transport in S. cerevisiae, a key biofuel cell factory. Hence, in this study, we focused on identifying efflux pumps that potentially transport alkane biofuels and harnessing those pumps as a direct mechanism for increasing tolerance through efflux pumping of alkanes from cells.

To this end, we considered ATP-binding cassette (ABC) transporters of Yarrowia lipolytica, an oleaginous yeast that efficiently assimilates and utilizes hydrophobic substrates such as alkanes, fatty acids and lipids [26-28]. Further, the mutants lacking part of $\mathrm{ABC}$ transporters show defective phenotypes for alkane utilization as a carbon source $[29,30]$. Consequently, in this study, we attempted to improve the tolerance of $S$. cerevisiae against alkanes using $Y$. lipolytica $\mathrm{ABC}$ transporters. Notably, we demonstrated that $\mathrm{ABC} 2$ and $\mathrm{ABC} 3$ transporters maintained 5 and 30-fold lower intracellular decane and undecane levels respectively, and significantly improved tolerance in S. cerevisiae, evidenced by about 80 -fold increase in the tolerance limit against decane.

\section{Results}

Transcription activity of $A B C$ transporters with alkanes To assess whether $\mathrm{ABC} 1, \mathrm{ABC} 2 \mathrm{ABC} 3$ and/or $\mathrm{ABC} 4$ transporters were involved in alkane transport in $Y$. lipolytica, we analysed the effects of alkanes with different chain lengths (C8-C12) on the transcription levels of these four $\mathrm{ABC}$ transporter genes using quantitative RT-PCR. The reason that $\mathrm{C} 8$ to $\mathrm{C} 12$ alkanes were tested in this study was that longer alkanes showed no toxicity toward S. cerevisiae (data not shown). Compared with control samples without alkane treatment, the transcription levels of $A B C 1$ and $A B C 4$ showed no change when the cells were treated with different alkanes (C8-C12) (Figure 1). However, the mRNA levels of $\mathrm{ABC} 2$ were significantly increased when $Y$. lipolytica was treated with octane (C8), nonane (C9), decane (C10) and undecane (C11) $(\mathrm{p}<0.05)$, while the mRNA levels of $\mathrm{ABC} 3$ were significantly increased toward nonane (C9) and decane (C10) $(p<0.05)$ (Figure 1). These results strongly suggested that two of the $\mathrm{ABC}$ transporters, $\mathrm{ABC} 2$ and $\mathrm{ABC} 3$, might play a critical role in the transport of alkanes for C8, C9, C10 and $\mathrm{C} 11$ alkanes. Thus, based on the qRT-PCR results, $\mathrm{ABC} 2$ and $\mathrm{ABC} 3$ were chosen for further analysis of their alkane transport capability.

\section{Expression and subcellular localization of $A B C 2$ and $A B C 3$}

To confirm the expression of these two transporters in S. cerevisiae, a $6 \mathrm{x}$ His tag was attached to the $\mathrm{C}$ terminus of $\mathrm{ABC} 2$ and $\mathrm{ABC}$. Through immunodetection of $6 \mathrm{x}$ His-tagged proteins, specific bands were assigned to ABC2 (165 kDa) and ABC3 (167 kDa) (Figure 2A). This western blot result confirmed the expression of $\mathrm{ABC} 2$ and $\mathrm{ABC} 3$ in S. cerevisiae. Next, to further analyse the localization of $\mathrm{ABC} 2$ and $\mathrm{ABC} 3$, each of them was tagged with EGFP at its $C$ terminus. As shown in Figure 2B, strong fluorescence was observed on the plasma membrane of cells containing ABC2-EGFP and ABC3-EGFP fusion proteins, respectively. These results suggest that $\mathrm{ABC} 2$ and $\mathrm{ABC} 3$ were expressed and located on the plasma membrane of $S$. cerevisiae.

To investigate whether EGFP would affect the localization of the transporters, the functionality of $A B C 2$ - and $\mathrm{ABC} 3$-EGFP was compared with that of $\mathrm{ABC} 2$ and $\mathrm{ABC} 3$. This functionality was determined by the viability of the cells in the presence of alkanes. The growth patterns of strains expressing $\mathrm{ABC} 2 / 3$-EGFP fusion protein and $\mathrm{ABC} 2 / 3$ were similar (data not shown). These data indicate that EGFP fusion proteins function similarly as the untagged proteins. Thus, EGFP tag does not affect the localization of the transporter proteins.

\section{$A B C 2$ and $A B C 3$ contribute to increasing alkane tolerance} After confirming that $\mathrm{ABC} 2$ and $\mathrm{ABC} 3$ were expressed on the cell membrane, we conducted susceptibility assays to study the effect of $\mathrm{ABC} 2$ and $\mathrm{ABC} 3$ on the tolerance of the cells toward alkanes. The toxic effects of alkanes on $S$. cerevisiae with $\mathrm{ABC} 2$ and $\mathrm{ABC} 3$ were measured through alkane susceptibility assays on agar plates. 

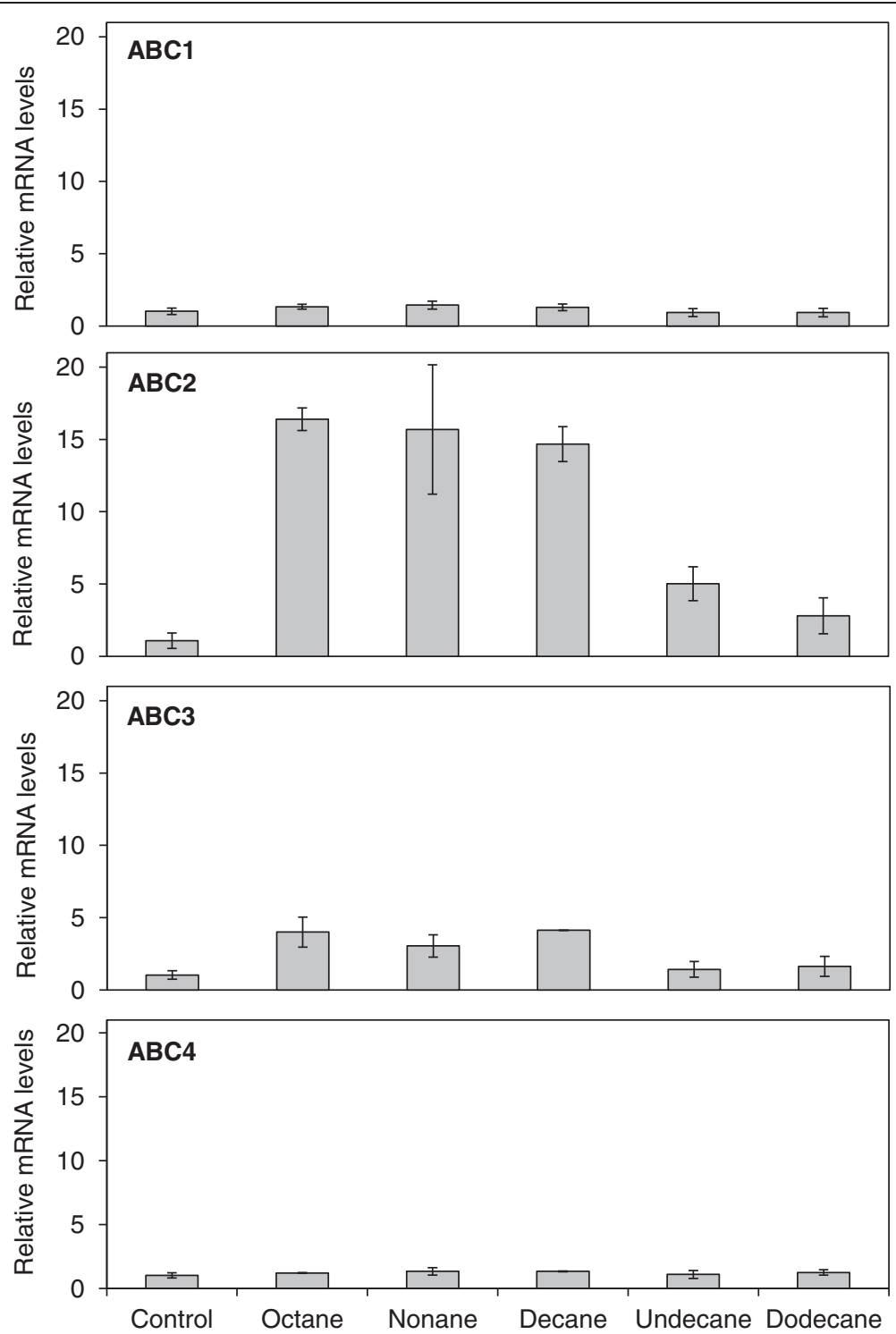

Figure 1 mRNA transcript levels of $A B C 1-4$ genes in $Y$. lipolytica. Quantitative RT-PCR analyses of $A B C 1, A B C 2, A B C 3$ and $A B C 4$ in $Y$. lipolytica cells upon treatment with octane, nonane, decane, undecane and dodecane (C8-C12). Each value of qRT-PCR was normalized to $\beta$-actin expression levels and expressed as the fold change relative to the levels detected in control samples, which were cells without alkane treatment and set equal to 1. Error bars represent the standard deviation (SD) of three biological replicates. Primers for qRT-PCR are listed in Table S1.

Figure 3A shows that octane, nonane, decane and undecane were toxic to $S$. cerevisiae cells. However, in cells expressing $\mathrm{ABC} 2$ and $\mathrm{ABC} 3$, cellular tolerance toward decane and undecane was considerably improved. It was observed that the expression of $\mathrm{ABC} 2$ led to higher tolerance toward decane than $\mathrm{ABC}$. Note that $\mathrm{ABC} 1$ and $\mathrm{ABC} 4$ expression led to no tolerance improvement (Additional file 1: Figure S1). Therefore, the results above suggest that $\mathrm{ABC} 2$ and $\mathrm{ABC} 3$ successfully improved alkane tolerance in S. cerevisiae.

To further examine quantitative effects of $\mathrm{ABC} 2$ and $\mathrm{ABC} 3$ toward decane and undecane, alkane susceptibility tests in liquid culture were conducted. As shown in
Figure 3B, the tolerance limit of S. cerevisiae was below $0.50 \%$ and $20 \%$ for decane and undecane, respectively. With $\mathrm{ABC} 2$ transporter, the tolerance limit was increased to over $20 \%$ for both decane and undecane. With $A B C 3$ transporter, the tolerance limit of $S$. cerevisiae was improved to over $0.75 \%$ and $20 \%$ for decane and undecane, respectively. This result suggests that the expression of $A B C 2$ and $A B C 3$ transporters significantly improved the tolerance toward decane and undecane.

To study the time-course behavior of $\mathrm{ABC} 2$ - and ABC3-expresssing $S$. cerevisiae cells in the presence of $0.5 \%$ decane and $20 \%$ undecane, which caused no growth in the wild-type cells, the growth patterns were studied. 
A

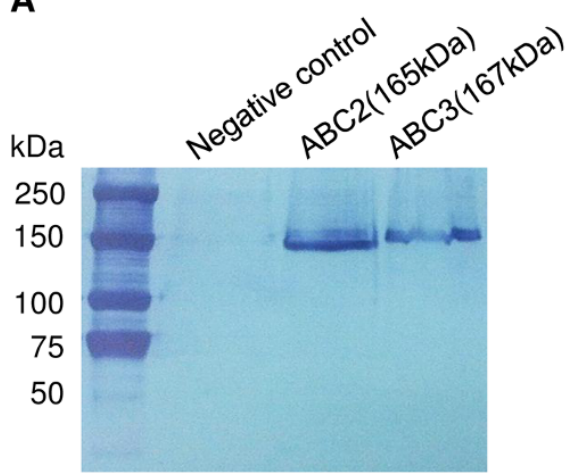

B

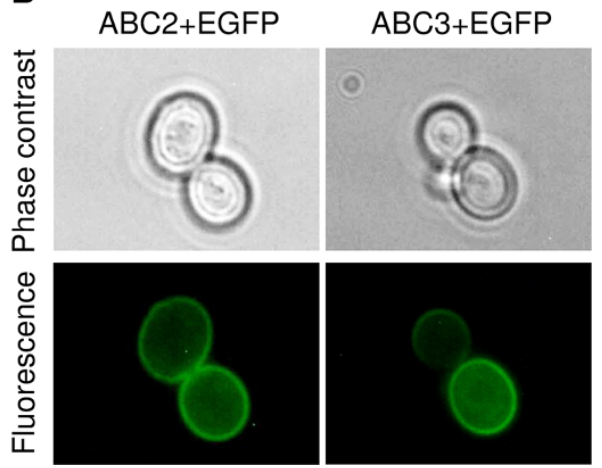

Figure 2 Expression and subcellular localization of $\mathbf{A B C}$ and $\mathbf{A B C}$. (A) Expression of $A B C 2$ and $A B C 3$. The expression of $A B C 2$ and $A B C 3$ carrying 6 xHis tag was confirmed by western blot analysis. The positions of molecular mass markers are indicated at left. Western blot analyses were performed as described under "Methods". (B) Subcellular localization of ABC2 and ABC3 determined by fluorescence microscopy. Yeast cells carrying plasmids encoding ABC2-EGFP or ABC3-EGFP fusion proteins were cultured and harvested. Phase contrast figures and fluorescence figures are shown.

As shown in Figure 3C, while S. cerevisiae cells without the transporters exhibited no growth, the cells with $\mathrm{ABC} 2$ and $\mathrm{ABC} 3$ transporters grew normally after initial growth delays. ABC3 expressing cells underwent a longer growth delay under both alkane treatments, but exhibited a slightly higher growth rate with decane treatment. Note that $S$. cerevisiae cells expressing $\mathrm{ABC} 2$ and $\mathrm{ABC} 3$ transporters exhibited similar growth rates in the absence of alkanes (Figure 3C, Additional file 1: Table S2).

\section{$A B C 2$ and $A B C 3$-mediated efflux pumping of alkanes}

The results of susceptibility assay on agar plates and in liquid medium showed that both $\mathrm{ABC} 2$ and $\mathrm{ABC} 3$ transporters greatly enhanced tolerance toward decane and undecane, with $\mathrm{ABC} 2$ leading to stronger tolerance. We therefore hypothesized that the tolerance generated by $\mathrm{ABC} 2$ and $\mathrm{ABC} 3$ was linked to the efflux pumping of decane and undecane. Alkane efflux pumping might help to maintain an intracellular alkane concentration under a toxic threshold.

To verify this hypothesis, intracellular alkane accumulation was analysed after $48 \mathrm{~h}$ incubation with $0.5 \%$ decane or $20 \%$ undecane. As shown in Figure 4, cells expressing $\mathrm{ABC} 2$ and $\mathrm{ABC} 3$ had $\sim 5$-fold lower intracellular decane level relative to the control. Further, $\mathrm{ABC} 2$ and $\mathrm{ABC} 3$ transporters were shown to reduce the intracellular undecane level approximately 30-fold compared to the control. The sharp decrease in intracellular alkane levels strongly suggests that $\mathrm{ABC} 2$ and $\mathrm{ABC} 3$ function as decane and undecane efflux pumps.

\section{Glutamate is required for energy-dependent efflux pumping of $A B C 2$ and $A B C 3$}

The main characteristic of $A B C$ transporters is that $A B C$ transporters utilize the energy of ATP hydrolysis to carry out biological processes. The ATP hydrolysis model is essential for $A B C$ transporters. Thus, to better understand $\mathrm{ABC} 2$ and $\mathrm{ABC} 3$ 's mechanisms in alkane export, we looked into the possible ATP hydrolysis models for both transporters. Two different models of ATP hydrolysis mechanisms were proposed for $\mathrm{ABC}$ transporters before: the "catalytic carboxylate" model [31] and the "catalytic dyad" model [32]. According to the "catalytic carboxylate" model, the highly conserved glutamate residue at the $C$ terminus of the Walker B motif is essential for ATP hydrolysis. However, in the "catalytic dyad" model, interactions between glutamate of the Walker B motif and the histidine of the $\mathrm{H}$-loop are a prerequisite for ATP hydrolysis.

Protein analyses revealed four possible locations of characteristic domains in $\mathrm{ABC}$ transporters, namely two nucleotide-binding domains (NBD1 \& 2) and two transmembrane domains (TMD1 \& 2) [33,34]. Sequence alignment of $\mathrm{ABC} 2, \mathrm{ABC} 3, \mathrm{PDR} 5$ and PDR15 showed that these proteins have high similarities in NBD domains which include Walker A motif, Walker B motif, C-loop and H-loop (Figure 5A). Similar to the widely studied PDR5 model [33,34], critical amino acids such as glutamate in C-terminus of Walker B motif and histidine of H-loop are only present in NBD2 but not in NBD1 for $\mathrm{ABC} 2$ and $\mathrm{ABC} 3$ [35].

Hence, to determine the ATP hydrolysis mechanism of $\mathrm{ABC} 2$ and $\mathrm{ABC} 3$, the glutamate (E988 for $\mathrm{ABC} 2$ and $\mathrm{E} 989$ for $A B C 3)$ and the histidine (H1020 for $A B C 2$ and $\mathrm{H} 1021$ for $\mathrm{ABC} 3$ ) in $\mathrm{NBD} 2$ of $\mathrm{ABC} 2$ and $\mathrm{ABC} 3$ were mutated to glutamine and alanine, respectively (Additional file 1: Figure S2). As shown in Figure 5B, ABC2-E988Q and ABC3-E989Q mutants were highly sensitive against both decane and undecane, while $\mathrm{ABC} 2-\mathrm{H} 1020 \mathrm{~A}$ and $\mathrm{ABC} 3-\mathrm{H} 1021 \mathrm{~A}$ 

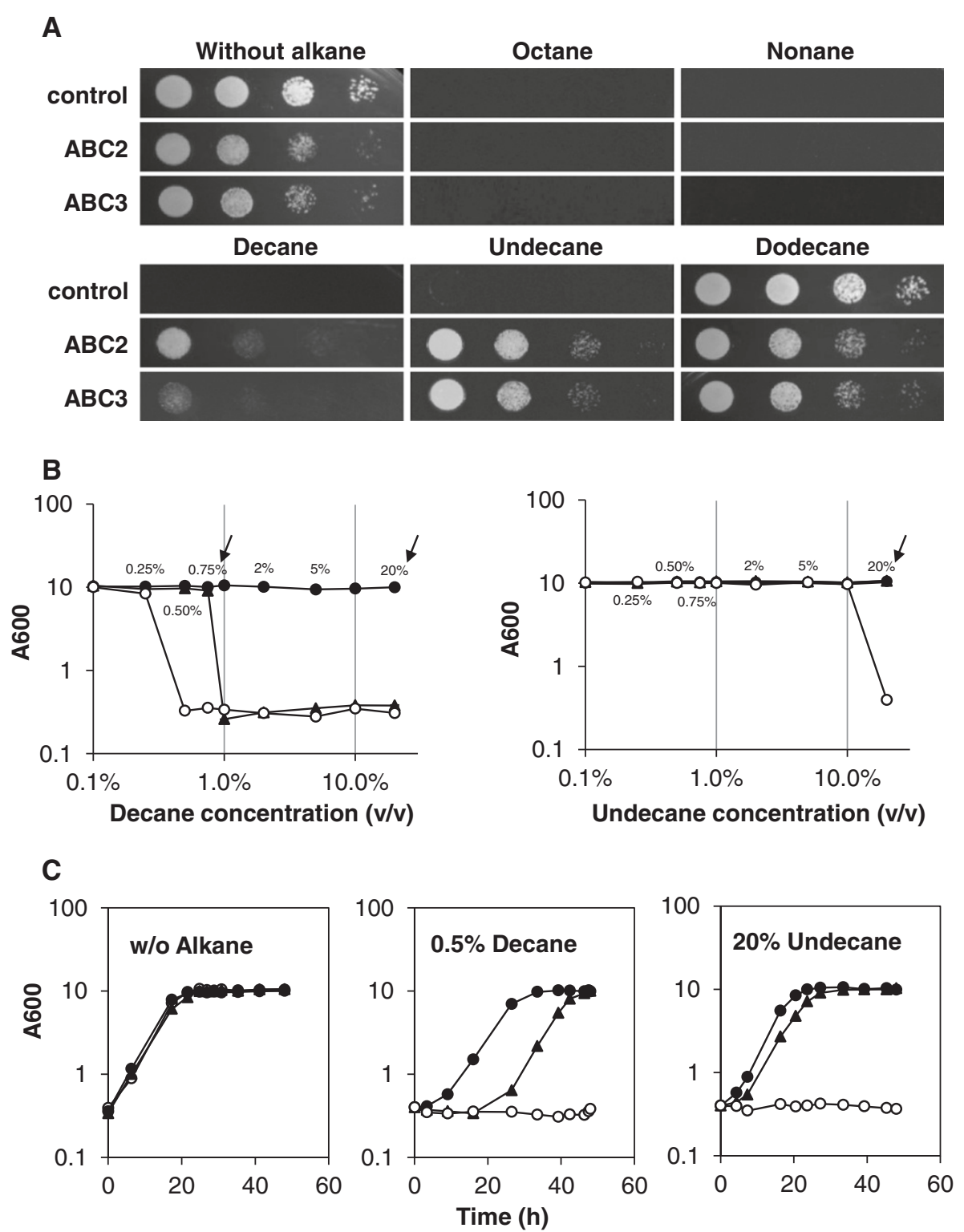

Figure 3 Alkane susceptibility assay for S. cerevisiae. Alkane susceptibility assays were performed in S. cerevisiae cells expressing ABC2, ABC3 or with an empty plasmid. (A) Alkane susceptibility assay on agar plates. Serial 10-fold dilutions (from left to right: non diluted, $10^{-1}, 10^{-2}, 10^{-3}$ ) of cells were spotted on agar plates with alkanes (octane, nonane, decane, undecane and dodecane) as vapor phase. Plates were incubated at $28^{\circ} \mathrm{C}$ for 2 days. (B) Alkane susceptibility assay in liquid culture. Overnight cell culture was diluted into induction medium (final $\mathrm{OD}_{600}=0.4$ ) with alkanes (decane or undecane). The cell culture was incubated for $48 \mathrm{~h}$ at $28^{\circ} \mathrm{C}$. The $\mathrm{OD}_{600}$ value of each sample was determined and plotted against its corresponding alkane concentration $(0.1 \%, 0.25 \%, 0.5 \%, 0.75 \%, 1 \%, 2 \%, 5 \%, 10 \%$ and $20 \% \mathrm{vol} / \mathrm{vol})$. Each point represents the mean of three biological replicates; standard deviations are presented. Arrows indicate increased cell tolerance towards alkanes. (C) Growth file of cells in alkaneinhibiting conditions $(0.5 \%$ ( $\mathrm{vol} / \mathrm{vol})$ decane or $20 \%$ (vol/vol) undecane). Symbols for strains are: control sample with empty plasmid (open circle), cells expressing $A B C 2$ (filled circle), cell expressing $A B C 3$ (filled triangle).

mutants still showed increased tolerance against undecane. Therefore, histidine is deemed not as essential as glutamate for these transporters' activity, and ATP is most likely to be hydrolyzed by the catalytic carboxylate mechanism.

\section{Discussion}

In biofuel production, end products such as alkanes are frequently toxic to host cells, thereby placing a limit on the yield. Thus, cellular tolerance optimization is essential in the production of biofuels. To improve 


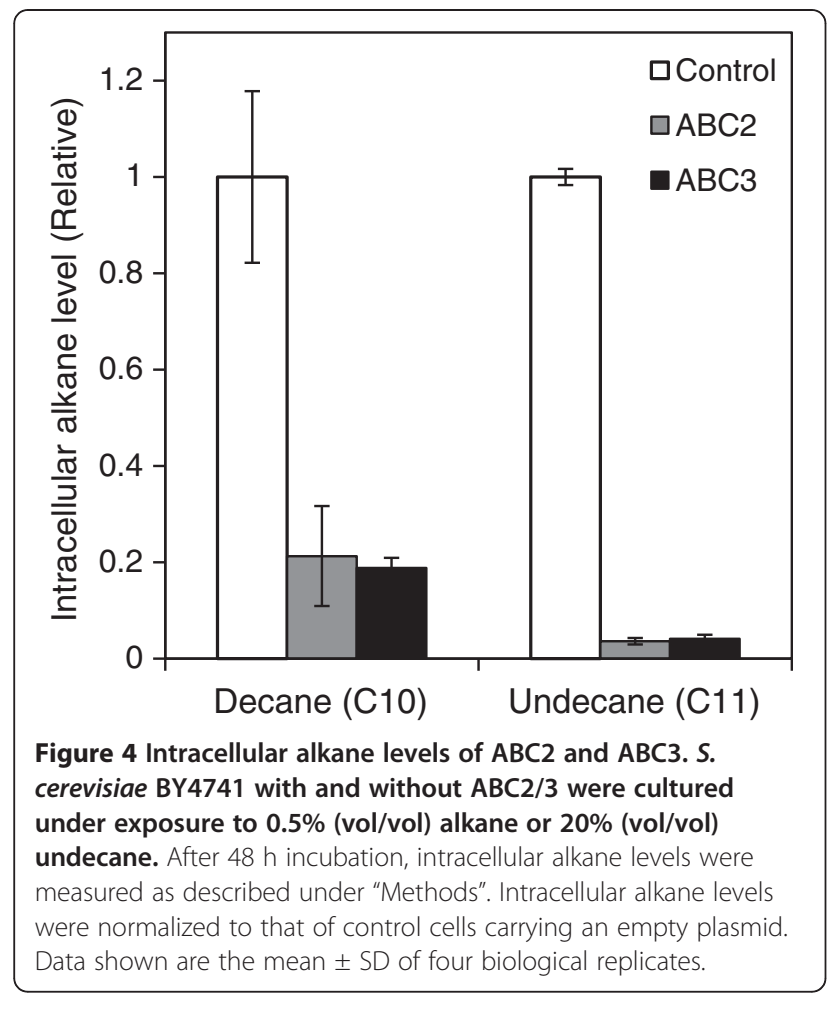

the yield and productivity, engineering strategies, including increasing tolerance toward biofuels in producing host cells, need to be developed. To this end, here, we focused on improving alkane tolerance in $S$. cerevisiae. Our alkane susceptibility assays revealed that $\mathrm{C} 12$ or longer alkanes exhibited little or no toxicity toward S. cerevisiae (data not shown), while C8C11 alkanes were toxic. This result suggests that longer chain length alkanes have lower cell toxicity toward S. cerevisiae, in agreement with the previous study by Gill and Ratledge (1972) [15] demonstrating that the toxicity of alkanes is related to their chain length. The proposed mechanisms for this phenomenon are as follows. First, higher chain lengths lead to increasing molecular weight. The higher the molecular weight is, the harder it is for the compound to penetrate yeast cell membrane. Second, a previous study [18] suggested that a compound whose $\log P_{\text {ow }}$ is between 1.5 and 6.0 is considered extremely toxic to microorganisms and other living cells, such as nonane $\left(\log P_{o w}=5.5\right)$ and decane $\left(\log P_{o w}=6.0\right)$. Higher chain length alkanes have higher $\log P_{o w}$ value. For example, C12 alkane has $\log P_{\text {ow }}$ of 7.0 and alkanes with chain length above 12 have higher $\log P_{\text {ow }}$. This reported correlation between toxicity and $\log P_{o w}$ is in line with our observation that alkanes with longer chain length ( $\mathrm{C} 12$ or longer) show insignificant toxicity toward S. cerevisiae. Since longer alkanes showed insignificant toxicity toward S. cerevisiae, this study was focused on improving the tolerance of $S$. cerevisiae against alkanes with $\mathrm{C} 12$ and below.

$\mathrm{ABC} 2$ and $\mathrm{ABC} 3$ were selected as candidates based on their potentials toward alkane efflux pumping. Although both $\mathrm{ABC} 2$ and $\mathrm{ABC} 3$ expressing cells showed increased $\mathrm{C} 10$ and $\mathrm{C} 11$ alkane tolerance, $\mathrm{ABC} 2$ exhibited better performance than $A B C 3$, suggesting that $A B C 2$ is a more effective pump for alkane transport. Thus, we looked into the possible reasons for the difference in efficiency between these two transporters. One plausible reason is the difference in kinetics of substrate-protein interactions and/or nucleotide-protein interactions between $\mathrm{ABC} 2$ and $\mathrm{ABC} 3$. For example, Low et al. (2010) [36] found that difference in TMD residues can lead to differences in $A B C$ transporter efficiency. It was hypothesized that the TMD residues might affect substrate affinity, resulting in differences in substrateprotein kinetics. Another reason for the different efflux efficiencies may be post-translational modification patterns. Protein phosphorylation was found to be essential for the stability of the yeast multidrug transporter Pdr5p [37]. As hypothesized before, transporters, such as Pdh1p that requires more phosphorylations than Cdr1p, may take longer time to reach its active, substrateeffluxing form [38]. Thus, similar to Pdh1p, it is possible that $\mathrm{ABC} 3$ requires longer phosphorylation time to arrive at its active alkane-effluxing form.

Besides the difference in efflux pumping efficiencies of $A B C 2$ and $A B C 3$, we also observed that $A B C 2$ and $\mathrm{ABC} 3$ did not increase tolerance toward shorter chain alkanes such as C8 and C9. Future work therefore may include directed evolution of these transporters to broaden their substrate specificity, mainly toward shorter chain alkanes. For example, random mutagenesis was shown to affect substrate specificity of $A B C$ transporters [39]. Also, protein structure studies may be needed for better understanding of the exact substrate binding and transport mechanism.

\section{Conclusions}

In this study, we demonstrated that transporter engineering through expression of heterologous efflux pumps led to improved tolerance against alkanes in S. cerevisiae, a key industrial microbial host. In particular, we showed that the tolerance limit of $S$. cerevisiae was increased about 80 -fold against decane. Further, we provided the evidence that the improved tolerance was primarily due to the lowering of the intracellular alkane level wherein $\mathrm{ABC} 2$ and $\mathrm{ABC} 3$ function as an efflux pump. To our knowledge, this is the first study to harness a transporter-engineering strategy for alkane tolerance improvement in eukaryotic host cells, which is readily applicable to alkane biofuel-producing microbes. We believe that our work here laid the groundwork for 


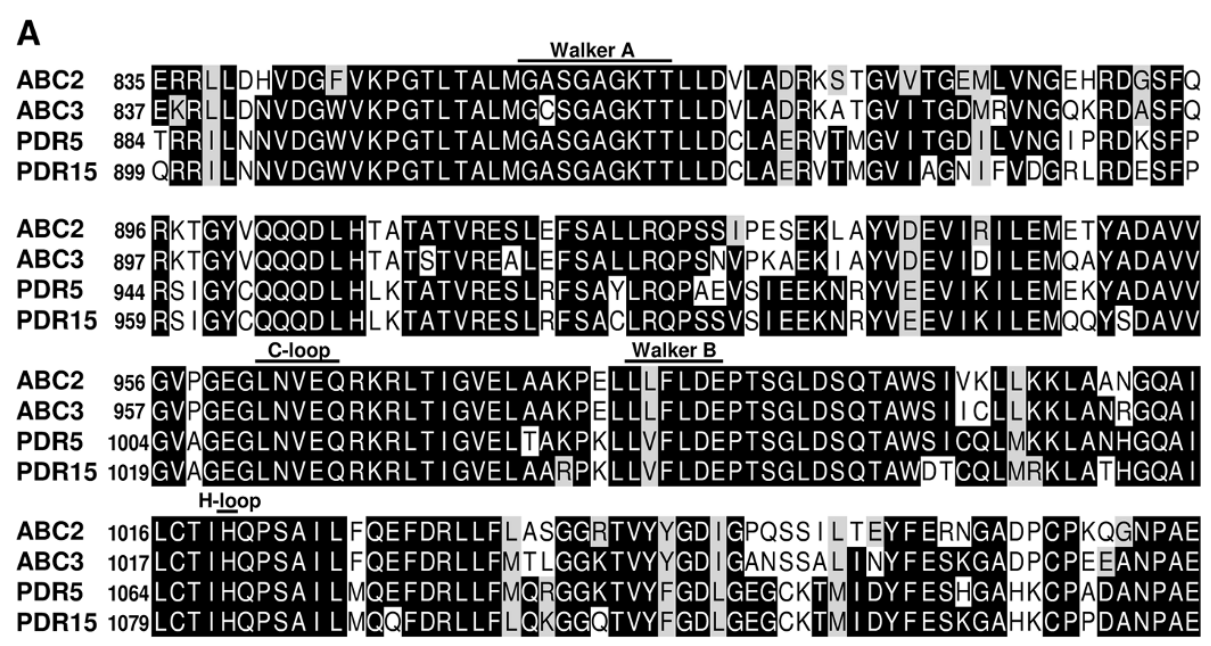

B

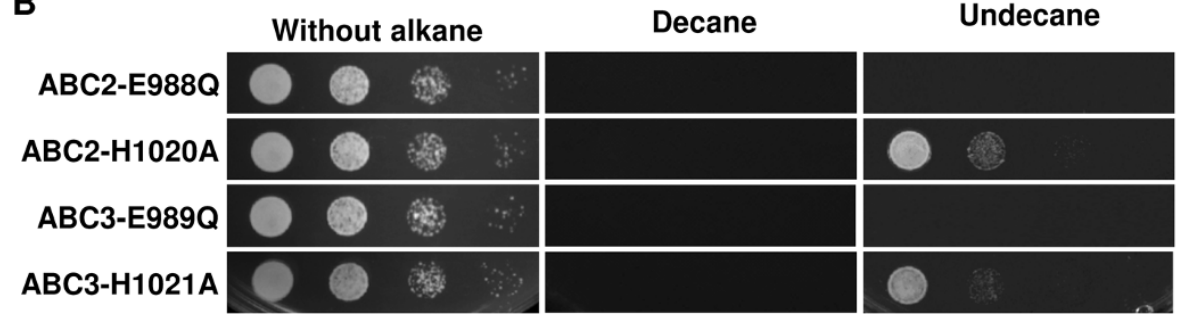

Figure 5 Transporter sequence comparison and alkane susceptibility assay of $A B C 2$ and $A B C 3$ mutants. (A) Multiple sequence alignment of NBD2 of ABC transporters. ABC2 [GenBank: CAG82364]; ABC3 [GenBank: CAG82646]; PDR5 [GenBank: CAA99359]; and PDR15 [GenBank: AAB64846]. Starting amino acid numbers are shown in each line. Sequences were aligned in ClustalW, and the shading was created by Mobyle Pasteur Boxshade. Single letter abbreviations for amino acid residues are as follows: A, Ala; C, Cys; D, Asp; E, Glu; F, Phe; G, Gly; H, His; I, lle; K, Lys; L, Leu; M, Met; N, Asn; P, Pro; Q, Gln; R, Arg; S, Ser; T, Thr; V, Val; W, Trp; and Y, Tyr. (B) Alkane susceptibility assay with ABC2 and ABC3 mutants. Susceptibility assays were performed as described under "Methods".

developing robust alkane-producing yeast cells through transporter engineering, which will greatly aid in nextgeneration alkane biofuel production and recovery.

\section{Methods}

Strains and media

All cells involved in cloning experiments were E. coli TOP10 (Invitrogen) unless otherwise stated. LuriaBertani (BD) was used as the medium for cloning studies unless otherwise stated. Ampicillin $(100 \mu \mathrm{g} / \mathrm{ml})$ was added to the culture media for antibiotic selection where appropriate.

The yeast strains S. cerevisiae BY4741 (ATCC 201388) and Y. lipolytica CLIB122 (CIRM) were used for function characterization. S. cerevisiae BY4741 was cultured in rich medium (YPD), synthetic minimal medium lacking uracil $(\mathrm{SC}-\mathrm{U})$ or induction medium. YPD medium (1\% yeast extract, $2 \%$ peptone and $2 \% \mathrm{D}$-glucose) was used to routinely maintain wild type strain. SC-U medium $(0.67 \%$ yeast nitrogen base, $0.192 \%$ uracil dropout and $2 \%$ raffinose) was used for growing pYES2 transformants. Induction medium $(0.67 \%$ yeast nitrogen base, $0.192 \%$ uracil dropout, $1 \%$ raffinose and $2 \%$ galactose) was used for protein induction in S. cerevisiae cells. Medium containing $0.67 \%$ yeast nitrogen base supplemented with $0.5 \%$ casein hydrolysate and $2 \%$ glucose was used for growth of $Y$. lipolytica for qRT-PCR sample preparation. Solid media were supplemented with $2 \%$ agar. Yeast growth media components were purchased from Sigma-Aldrich.

Alkanes (octane (C8), nonane (C9), decane (C10), undecane $(\mathrm{C} 11)$ and dodecane $(\mathrm{C} 12)$ ) purchased from Sigma-Aldrich were added to culture media for protein function analysis where appropriate.

\section{Plasmid construction}

A list of the oligonucleotides used is shown in Additional file 1: Table S1. Plasmid pYES2 (Invitrogen) with the GAL1 promoter was used as an expression vector.

To clone 6x His-tagged ABC2, genomic DNA of $Y$. lipolytica CLIB122 was used as a PCR template with two pairs of primers $A B C 2-F 1 / A B C 2-R 1$, and $A B C 2-F 2$ /ABC2-R2. The two PCR products were combined through the Splicing Overlap Extension (SOE) method [40] using flanking primers $\mathrm{ABC} 2-\mathrm{F} 1$ and $\mathrm{ABC} 2-\mathrm{R} 2$. The resulting DNA fragment was digested with HindIII and 
NotI and cloned into pYES2 cut with the same restriction enzymes, creating pYES2ABC2. Plasmid pYE$\mathrm{S} 2 \mathrm{ABC} 3$ was constructed as for pYES2ABC2. Sitedirected mutagenesis of the transporters ABC2-E988Q, ABC2-H1020A, ABC3-E989Q and ABC3-H1021A were constructed by mutating glutamate to glutamine and histidine to alanine respectively.

Plasmid pYES2ABC2-EGFP, which encodes yeast enhanced green fluorescent protein (EGFP) at the Cterminus of the $\mathrm{ABC} 2$ open reading frame, was constructed as follows. We used pYES2ABC2 as a PCR template with primer set ABC2-F1 and ABC2-EGFP-R2. The resulting DNA fragment was digested with HindIII and NotI and cloned into pYES2 cut with the same restriction enzymes, creating pYES2ABC2-1. EGFP was amplified from pKT127 (Euroscarf) [41] using primer set EGFP-F and EGFP-R, digested with NotI and SphI and inserted into the same restriction sites of pYES2ABC2-1 to create pYES2ABC2-EGFP. Plasmid pYES2ABC3-EGFP was constructed as for pYES2ABC2EGFP. For construction of pYES2EGFP, EGFP was amplified by PCR from pKT127 using primers EGFP-control -F and EGFP-R, digested with NotI and SphI and cloned into pYES2. All restriction and ligation enzymes were purchased from New England Biolabs (NEB).

\section{Quantitative RT-PCR}

Total RNA samples from Y. lipolytica CLIB122 cells, which were treated and untreated with alkane for $24 \mathrm{~h}$, were prepared using RNeasy Mini Kit (Qiagen), followed by cDNA synthesis using $\mathrm{H}$ minus Reverse transcriptase kit (Fermentas). Quantitative RT-PCR analysis was performed on the Bio-Rad iQ5 real-time PCR detection system using SsoFast EvaGreen Supermix kit (Bio-Rad). The actin gene (YALI0D08272g) [42] was used as reference gene for $Y$. lipolytica. Relative mRNA levels were derived using comparative $\mathrm{C}_{\mathrm{T}}$ method. PCR primer sequences are listed in Additional file 1: Table S1.

\section{Western blot analysis}

S. cerevisiae cells carrying the plasmids encoding the $6 \mathrm{x}$ His-tagged $\mathrm{ABC} 2$ and $\mathrm{ABC} 3$ were cultured in induction medium and harvested at $\mathrm{OD}_{600}=1-2$ (early exponential phase). The protein extraction method here is based on alkaline lysis [43] and glass bead lysis [44] methods. The following handling process was carried out in the cold room $\left(\sim 4^{\circ} \mathrm{C}\right)$. Cell pellets (around $14 \mathrm{mg}$ ) were resuspended in $300 \mu \mathrm{l}$ cold lysis buffer $(0.1 \mathrm{M} \mathrm{NaOH}, 2 \%$ $\beta$-mercaptoethanol, and protease inhibitor mixture (Roche Applied Science)). After $5 \mathrm{~min}$, glass beads (425$600 \mu \mathrm{m}$, Sigma) were added to the suspension. The cells were lysed by vortexing for $2 \mathrm{~min}$. The lysate obtained was clarified by transferring supernatant into a new tube. Proteins in the lysate were fully dissolved by adding SDS (final concentration around 2\%) and gently stirring for $10 \mathrm{~min}$. After centrifugation, the supernatant was mixed equally with Laemmli sample buffer (Bio-Rad) and separated on a SDS-polyacrylamide gel. The sample gels were used for blotting. Proteins were blotted onto a $0.2 \mu \mathrm{m}$ nitrocellulose membrane (Bio-Rad) through Trans-Blot Turbo Blotting System (Bio-Rad). 6x Histagged $\mathrm{ABC} 2$ and $\mathrm{ABC} 3$ were detected using anti-6x His-tag antibody (HRP) (ab1187, Abcam) and 3,3',5,5'Tetramethylbenzidine (TMB) liquid substrate system (Sigma).

\section{Fluorescence microscopy}

S. cerevisiae BY4741 cells carrying plasmid pYES2ABC2EGFP or pYES2ABC3-EGFP were grown to the early exponential phase in induction medium, harvested and mounted on the poly-L-lysine-coated slide glass. EGFP fluorescence was analysed with a fluorescent microscope (Zeiss Axio Scope A1).

\section{Alkane susceptibility assays}

Alkane susceptibility test on agar plate: Alkane susceptibility test on plates was performed according to the methods of Mauersberger et al. [28,29]. Exponentially growing cells in induction medium were centrifuged and re-suspended until $\mathrm{OD}_{600}$ reaches 1 . Ten microliter aliquots of successive 10-fold dilutions (non diluted, $10^{-1}$, $10^{-2}, 10^{-3}$ ) of the cells were spotted onto the induction medium plate. Alkanes were supplied as vapour phase by placing $200 \mu \mathrm{l}$ alkane on a sterile filter paper in the lid of the petri dish. Plates were incubated at $28^{\circ} \mathrm{C}$ for 2 days.

Alkane susceptibility test in liquid culture: Overnight cultures were diluted into $5 \mathrm{ml}$ induction medium in $50 \mathrm{ml}$ glass bottle (Sigma) at an initial $\mathrm{OD}_{600}$ of 0.4 . Alkanes were added at different final concentrations. Bottles were sealed tightly with butyl rubber stopper (Sigma) and silver aluminum seal (Sigma). Liquid culture was performed at $28^{\circ} \mathrm{C}$ with shaking. Growth was monitored by measuring the $\mathrm{OD}_{600}$ at different time points. Cell cultures used for time point $\mathrm{OD}_{600}$ checking were collected from the glass bottle using needles and syringes.

\section{Determination of intracellular alkane levels}

After induction for $48 \mathrm{~h}$ with or without addition of alkanes, $S$. cerevisiae cells transformed with pYES2, pYES2ABC2 and pYES2ABC3 were harvested at $6000 \mathrm{~g}$ for $5 \mathrm{~min}$ at $4^{\circ} \mathrm{C}$. After washing with $50 \mathrm{mM}$ Tris.Cl, the cells were equally divided into two parts, one part for intracellular alkane extraction and the other for determination of total protein content. For alkane extraction, cell pellets were re-suspended in freshly prepared HPLC grade Chloroform/Methanol (v/v, 2:1). Dodecane was 
added into cell suspension as an internal standard. Acidwashed glass beads were added until the suspension was covered. Cells were then lysed by mechanical agitation using FastPrep-24 (MPBio) for $6 \mathrm{~min}$ at $6 \mathrm{~m} / \mathrm{s}$. The crude extract was obtained by pipettes. After addition of autoclaved $\mathrm{dd}_{2} \mathrm{O}$, the crude extract was emulsified for 10 min by inversion. After centrifugation, the crude extract was separated into two phases. The bottom phase containing alkane was transferred into a new $1.5 \mathrm{ml}$ microcentrifuge tube and purified as above with chloroform and $\mathrm{ddH} 2 \mathrm{O}$ until particulate matter was no longer observed. The purified solution was transferred into a clear GC vial for GC analysis. To check the total protein content, the cell pellets were re-suspended into $50 \mathrm{mM}$ Tris.Cl and lysed via mechanical agitation with acidwashed glass beads. Protein content of obtained crude extract was determined using the Bradford protein assay (Bio-Rad). Intracellular alkane levels were normalized to internal standard and cell lysate protein content $[45,46]$.

\section{Additional file}

Additional file 1: Table S1. Primers used in this study. Restriction sites are bold. Table S2. Specific growth rates under C10 and C11 alkane treatments. Figure S1. Alkane susceptibility assay with $A B C 1$ and $A B C 4$. Serial 10-fold dilutions (from left to right: non diluted, 10-1, 10-2, 10-3) of cells were spotted on agar plates with alkanes (octane, nonane, decane, undecane and dodecane) as vapor phase. Figure S2. To gengerate a topology model of $A B C 2(A)$ and $A B C 3(B)$, the TMHMMfix algorithm was used (http://www.sbc.su.se/ melen/TMHMMfix/). the resulting model was visualized by the TOPO2 transmembrane protein display software (http://www.sacs.ucsf.edu/cgi-bin/open-topo2.py). The Walker A motifs are shown in blue, the Walker B motifs are shown in green, and the C-loop are shown in orange. Residues that were mutated in this study are shown in red.

\section{Competing interests}

The authors declared competing financial interests.

\section{Authors' contribution}

$\mathrm{BC}$ and MWC conceived the project, designed the experiments, and wrote the manuscript. BC and HL conducted the experiments and analyzed the data. MWC supervised the project. All authors read and approved the final manuscript.

\section{Acknowledgements}

This work was supported by the Competitive Research Programme of the National Research Foundation of Singapore (NRF-CRP5-2009-03).

Received: 18 October 2012 Accepted: 8 February 2013

Published: 13 February 2013

\section{References}

1. Matsushika A, Watanabe S, Kodaki T, Makino K, Sawayama S: Bioethanol production from xylose by recombinant Saccharomyces cerevisiae expressing xylose reductase, NADP(+)-dependent xylitol dehydrogenase, and xylulokinase. J Biosci Bioeng 2008, 105(3):296-299.

2. Steen EJ, Chan R, Prasad N, Myers S, Petzold CJ, Redding A, Ouellet M, Keasling JD: Metabolic engineering of Saccharomyces cerevisiae for the production of n-butanol. Microb Cell Fact 2008, 7:36.

3. Kalscheuer R, Luftmann H, Steinbuchel A: Synthesis of novel lipids in saccharomyces cerevisiae by heterologous expression of an unspecific bacterial acyltransferase. Appl Environ Microbiol 2004, 70(12):7119-7125.
4. Yu KO, Jung J, Kim SW, Park CH, Han SO: Synthesis of FAEEs from glycerol in engineered Saccharomyces cerevisiae using endogenously produced ethanol by heterologous expression of an unspecific bacterial acyltransferase. Biotechnol Bioeng 2012, 109(1):110-115.

5. Shi S, Valle-Rodriguez JO, Khoomrung S, Siewers V, Nielsen J: Functional expression and characterization of five wax ester synthases in Saccharomyces cerevisiae and their utility for biodiesel production. Biotechnol Biofuels 2012, 5(1):7.

6. de Jong B, Siewers V, Nielsen J: Systems biology of yeast: enabling technology for development of cell factories for production of advanced biofuels. Curr Opin Biotechnol 2012, 23(4):624-630.

7. Gong P-F, Xu J-H: Bio-resolution of a chiral epoxide using whole cells of Bacillus megaterium ECU1001 in a biphasic system. Enzyme Microb Technol 2005, 36(2-3):252-257.

8. Klibanov AM: Improving enzymes by using them in organic solvents. Nature 2001, 409(6817):241-246.

9. Dembitsky VM, Srebnik M: Variability of hydrocarbon and fatty acid components in cultures of the filamentous cyanobacterium scytonema $\mathrm{sp}$. Isolated from microbial community "black cover" of limestone walls in Jerusalem. Biochem (Mosc) 2002, 67(11):1276-1282.

10. Schirmer A, Rude MA, Li X, Popova E, del Cardayre SB: Microbial biosynthesis of alkanes. Science 2010, 329(5991):559-562.

11. Tillman JA, Seybold SJ, Jurenka RA, Blomquist GJ: Insect pheromones-an overview of biosynthesis and endocrine regulation. Insect Biochem $\mathrm{Mol}$ Biol 1999, 29(6):481-514.

12. Samuels $L$, Kunst $L$, Jetter R: Sealing plant surfaces: cuticular wax formation by epidermal cells. Annu Rev Plant Biol 2008, 59:683-707.

13. Harger M, Zheng L, Moon A, Ager C, An JH, Choe C, Lai Y-L, Mo B, Zong D, Smith MD, et al: Expanding the product profile of a microbial alkane biosynthetic pathway. ACS Synthetic Biol 2013, 2(1):59-62.

14. Bernard A, Domergue F, Pascal S, Jetter R, Renne C, Faure J-D, Haslam RP, Napier JA, Lessire R, Joubès J: Reconstitution of Plant Alkane Biosynthesis in Yeast Demonstrates That Arabidopsis ECERIFERUM1 and ECERIFERUM3 Are Core Components of a Very-Long-Chain Alkane Synthesis Complex. Online: The Plant Cell; 2012.

15. Gill CO, Ratledge C: Toxicity of n-Alkanes, n-Alk-1-enes, n-Alkan-1-ols and n-Alkyl-1-bromides towards Yeasts. J Gen Microbiol 1972, 72(1):165-172.

16. Ramos JL, Duque E, Gallegos MT, Godoy P, Ramos-Gonzalez MI, Rojas A, Teran W, Segura A: Mechanisms of solvent tolerance in gram-negative bacteria. Annu Rev Microbiol 2002, 56:743-768.

17. Inoue A, Horikoshi K: A Pseudomonas thrives in high concentrations of toluene. Nature 1989, 338(6212):264-266.

18. Kawamoto T, Kanda T, Tanaka A: Preparation of an organic solventtolerant strain from baker's yeast. Appl Microbiol Biotechnol 2001, 55(4): 476-479.

19. Alper H, Moxley J, Nevoigt E, Fink GR, Stephanopoulos G: Engineering yeast transcription machinery for improved ethanol tolerance and production. Science 2006, 314(5805):1565-1568.

20. Jarboe LR, Grabar TB, Yomano LP, Shanmugan KT, Ingram LO: Development of ethanologenic bacteria. Adv Biochem Eng Biotechnol 2007, 108:237-261.

21. Dunlop MJ, Dossani ZY, Szmidt HL, Chu HC, Lee TS, Keasling JD, Hadi MZ, Mukhopadhyay A: Engineering microbial biofuel tolerance and export using efflux pumps. Mol Syst Biol 2011, 7:487.

22. Stanley D, Fraser S, Chambers PJ, Rogers P, Stanley GA: Generation and characterisation of stable ethanol-tolerant mutants of Saccharomyces cerevisiae. J Ind Microbiol Biotechnol 2010, 37(2):139-149.

23. Hou L: Improved production of ethanol by novel genome shuffling in Saccharomyces cerevisiae. Appl Biochem Biotechnol 2010, 160(4):1084-1093.

24. Kang A, Chang MW: Identification and reconstitution of genetic regulatory networks for improved microbial tolerance to isooctane. $\mathrm{Mol}$ Biosyst 2012, 8(4):1350-1358.

25. Teixeira M, Godinho C, Cabrito T, Mira N, Sá-Correia I: Increased expression of the yeast multidrug resistance $A B C$ transporter Pdr18 leads to increased ethanol tolerance and ethanol production in high gravity alcoholic fermentation. Microb Cell Fact 2012, 11(1):1-9.

26. Beopoulos A, Chardot T, Nicaud JM: Yarrowia lipolytica: A model and a tool to understand the mechanisms implicated in lipid accumulation. Biochimie 2009, 91(6):692-696.

27. Barth G, Gaillardin C: Physiology and genetics of the dimorphic fungus Yarrowia lipolytica. FEMS Microbiol Rev 1997, 19(4):219-237. 
28. Mauersberger S, Wang HJ, Gaillardin C, Barth G, Nicaud JM: Insertional Mutagenesis in the $n$-Alkane-Assimilating Yeast Yarrowia lipolytica: Generation of Tagged Mutations in Genes Involved in Hydrophobic Substrate Utilization. J Bacterio/ 2001, 183(17):5102-5109.

29. Thevenieau F, Le Dall MT, Nthangeni B, Mauersberger S, Marchal R, Nicaud JM: Characterization of Yarrowia lipolytica mutants affected in hydrophobic substrate utilization. Fungal Genet Biol 2007, 44(6):531-542.

30. Thevenieau F, Beopoulos A, Desfougeres T, Sabirova J, Albertin K, Zinjarde S, Nicaud JM: Uptake and Assimilation of Hydrophobic Substrates by the Oleaginous Yeast Yarrowia lipolytica. In Handbook of Hydrocarbon and Lipid Microbiology. Edited by Kenneth N. Berlin Heidelberg: Timmis. SpringerVerlag; 2010:1513-1527.

31. Moody JE, Millen L, Binns D, Hunt JF, Thomas PJ: Cooperative, ATPdependent association of the nucleotide binding cassettes during the catalytic cycle of ATP-binding cassette transporters. J Biol Chem 2002, 277(24):21111-21114.

32. Zaitseva J, Jenewein S, Jumpertz T, Holland IB, Schmitt L: H662 is the linchpin of ATP hydrolysis in the nucleotide-binding domain of the $A B C$ transporter HlyB. EMBO J 2005, 24(11):1901-1910.

33. Schmitt $L$, Tampé R: Structure and mechanism of $A B C$ transporters. Curr Opin Struct Biol 2002, 12(6):754-760.

34. Rutledge RM, Esser L, Ma J, Xia D: Toward understanding the mechanism of action of the yeast multidrug resistance transporter Pdr5p: a molecular modeling study. J Struct Bio/ 2011, 173(2):333-344.

35. Ernst R, Kueppers P, Klein CM, Schwarzmueller T, Kuchler K, Schmitt L: A mutation of the $\mathrm{H}$-loop selectively affects rhodamine transport by the yeast multidrug ABC transporter Pdr5. Proc Natl Acad Sci USA 2008, 105(13):5069-5074.

36. Low KO, Mahadi NM, Abdul Rahim R, Rabu A, Abu Bakar FD, Abdul Murad AM, Illias RM: Enhanced secretory production of hemolysin-mediated cyclodextrin glucanotransferase in Escherichia coli by random mutagenesis of the ABC transporter system. J Biotechnol 2010, 150(4):453-459.

37. Decottignies A, Owsianik G, Ghislain M: Casein Kinase I-dependent Phosphorylation and Stability of the Yeast Multidrug Transporter Pdr5p. J Biol Chem 1999, 274(52):37139-37146.

38. Wada S, Niimi M, Niimi K, Holmes AR, Monk BC, Cannon RD, Uehara Y: Candida glabrata ATP-binding cassette transporters Cdr1p and Pdh1p expressed in a Saccharomyces cerevisiae strain deficient in membrane transporters show phosphorylation-dependent pumping properties. $J$ Biol Chem 2002, 277(48):46809-46821.

39. Egner R, Rosenthal FE, Kralli A, Sanglard D, Kuchler K: Genetic Separation of FK506 Susceptibility and Drug Transport in the Yeast Pdr5 ATP-binding Cassette Multidrug Resistance Transporter. Mol Biol Cell 1998, 9(2):523-543.

40. Heckman KL, Pease LR: Gene splicing and mutagenesis by PCR-driven overlap extension. Nat Protoc 2007, 2(4):924-932.

41. Sheff MA, Thorn KS: Optimized cassettes for fluorescent protein tagging in Saccharomyces cerevisiae. Yeast 2004, 21(8):661-670.

42. Blanchin-Roland S, Da Costa G, Gaillardin C: ESCRT-I components of the endocytic machinery are required for Rim101-dependent ambient $\mathrm{pH}$ regulation in the yeast Yarrowia lipolytica. Microbiology 2005, 151(Pt 11):3627-3637.

43. Riezmant H, Hase T, Loon APGM, Grivell LA, Suda K, Schatz G: Import of proteins into mitochondria: a $70 \mathrm{kDa}$ outer membrane protein with a large carboxy-terminal deletion is still transported to the outer membrane. European Mol Biol Organ J 1983, 2(12):2161-2168.

44. Conzelmann A, Riezman H, Desponds C, Bron C: A major 125-kd membrane glycoprotein of Saccharomyces cerevisiae is attached to the lipid bilayer through an inositol-containing phospholipid. European $\mathrm{Mol}$ Biol Organ J 1988, 7(7):2233-2240.

45. Ramaswamy G, Karim MA, Murti KG, Jackowski S: PPARa controls the intracellular coenzyme A concentration via regulation of PANK1a gene expression. J Lipid Res 2004, 45(1):17-31.

46. Oda T, Hirota K, Nishi K, Takabuchi S, Oda S, Yamada H, Arai T, Fukuda K, Kita T, Adachi T, et al: Activation of hypoxia-inducible factor 1 during macrophage differentiation. Am J Physiol Cell Physiol 2006, 291(1):C104-C113.

\section{doi:10.1186/1754-6834-6-21}

Cite this article as: Chen et al:: Transporter engineering for improved

tolerance against alkane biofuels in Saccharomyces cerevisiae.

Biotechnology for Biofuels 2013 6:21.

\section{Submit your next manuscript to BioMed Central and take full advantage of:}

- Convenient online submission

- Thorough peer review

- No space constraints or color figure charges

- Immediate publication on acceptance

- Inclusion in PubMed, CAS, Scopus and Google Scholar

- Research which is freely available for redistribution 